\title{
Full-automatic Special Drill Hydraulic System and PLC Control
}

\author{
Xue Jun TIAN
}

Lingnan Normal University Electromechanical Engineering Research Institution Zhanjiang GuangdongChina

\begin{abstract}
A hydraulic-driven and PLC full-automatic special drill is introduced, working principle of the hydraulic system and PLC control system are analyzed and designed, this equipment has the advantages of high efficiency, superior quality and low cost etc.
\end{abstract}

Keywords: Full automatic special machine tool; Hydraulic system; PLC

\section{Introduction}

A full-automatic special drill consists of pedestal, workbench, spindle box, gearbox, cooling pump, rocker arm, drill and hopper etc. It can fulfill the one-off process from feeding, clamping to drilling as well as volume production. The whole operation process is described as follows: place the workpiece in the hopper, press the start button and the drill will work automatically; then take out the workpiece $a^{1}$ fter the drilling process is finished, the process of which not only greatly improves the work efficiency and the drilling quality but also relieves workers out of fatigue resulting from repeatedly carrying the parts. Compared with common drills, full-automatic special drill can realize process automation. The whole machining process is stable, secure and efficient because the hydraulic system features strong clamp force.

\section{Full-automatic special drill hydraulic system}

Working station structure of full-automatic special drill is shown in Fig. 1.

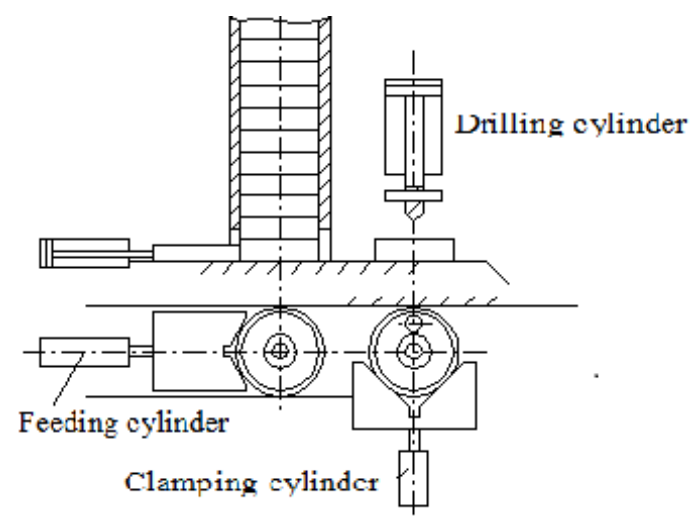

Fig. 1 Working station structure chart of full-automatic special drill

The working process is as follows: press the button $\rightarrow$ the feeding cylinder advances $\rightarrow$ the feeding cylinder starts to retreats $\rightarrow$ The feeding cylinder resets and the clamping cylinder advances $\rightarrow$ the drilling cylinder advances quickly $\rightarrow$ the drilling cylinder advances $\rightarrow$ the drilling cylinder retreats quickly $\rightarrow$ the clamping cylinder retreats $\rightarrow$ the feeding cylinder advances, repeatedly.

2 According to the above working process, selection of the reversing valve is analyzed below: the feeding cylinder is non-interfering with other cylinders, a two-position four-way solenoid valve can meet the feeding requirements; the clamping cylinder maintains certain pressure in the working process; if a two-position four-way magnetic exchange valve is selected, the pressure cannot be maintained when the drilling cylinder advances quickly and retreats quickly, so a 
three-position four-way solenoid valve is used; a three-position four-way solenoid valve is used for reversing of the drilling cylinder. A two-position two-way solenoid valve instead of the medium function of the reversing valve can used for unloading due to mutual interference between cylinders. Besides, To ensure stable operation, hydraulic cylinder metering-out control loop is used due to vertical installation of the drilling cylinder. Sequential action of three cylinders is controlled by travel switch and solenoid valve ${ }^{[1]}$. Full-automatic special drill hydraulic system loop is shown in Fig. 2, electric control analysis in Fig. 3 and electric control loop in Fig. 4.

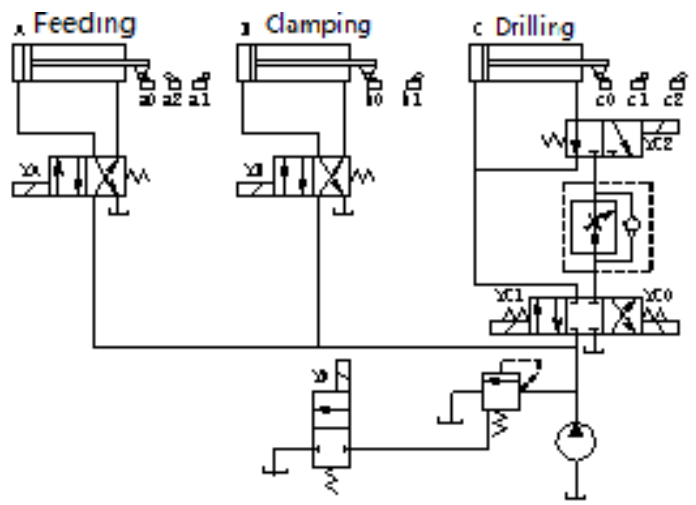

Fig. 2 Full-automatic special drill hydraulic system loop

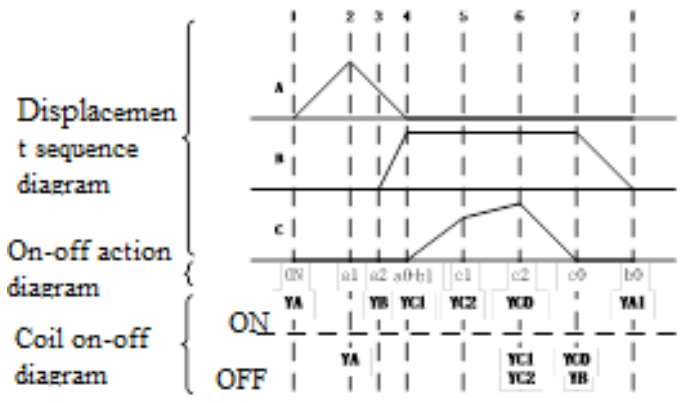

Fig. 3 Full-automatic special drill electric control analysis chart

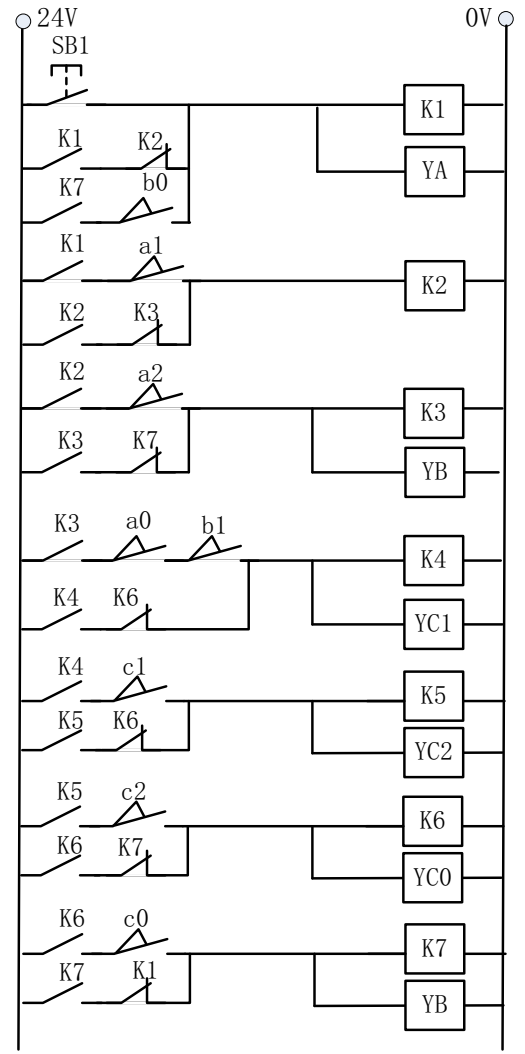

Fig. 4 Full-automatic special drill electric control loop diagram

(1) The feeding cylinder advances:

Press the switch and the feeding cylinder starts to work.

Inlet oil line: oil tank $\rightarrow$ oil hydraulic pump $\rightarrow($ YA electromagnet is powered on, the reversing valve changes the direction) $\rightarrow$ left cavity of the feeding cylinder;

Return oil line: left cavity of the feeding cylinder $\rightarrow$ reversing valve $\rightarrow$ oil tank.

(2) Movement of the clamping cylinder:

When the feeding cylinder piston rod moves to a2, YB solenoid valve is powered on and the clamping cylinder stays still; when the piston rod moves to al and the clamping cylinder extends, the feeding cylinder withdraws.

Inlet oil line of the feeding cylinder: oil tank $\rightarrow$ oil hydraulic pump $\rightarrow$ (YA electromagnet is powered off, the reversing valve changes the direction) $\rightarrow$ left cavity of the feeding cylinder;

Return oil line of the feeding cylinder: left cavity of the feeding cylinder $\rightarrow$ reversing valve $\rightarrow$ oil tank;

Inlet oil line of the clamping cylinder: oil tank $\rightarrow$ oil hydraulic pump $\rightarrow$ (YA electromagnet is powered on, the reversing valve changes the direction) $\rightarrow$ left cavity of the 
clamping cylinder;

Return oil line of the clamping cylinder: left cavity of the clamping cylinder $\rightarrow$ reversing valve $\rightarrow$ oil tank.

(3) Movement of the drilling cylinder:

When the feeding cylinder piston rod returns to a0 and the clamping cylinder moves to $\mathrm{b} 1, \mathrm{YC} 1$ is powered on and the drilling cylinder extends; then the drilling cylinder is under differential connection and moves quickly.

Inlet oil line of the drilling cylinder: oil tank $\rightarrow$ oil hydraulic pump $\rightarrow$ (YC1 electromagnet is powered on, the reversing valve changes the direction) $\rightarrow$ overflow valve $\rightarrow$ left cavity of the drilling cylinder;

Return oil of the drilling cylinder: right cavity of the drilling cylinder $\rightarrow$ left cavity of the drilling cylinder (the drilling cylinder is under differential connection);

Movement of the feeding cylinder and the clamping cylinder remains unchanged.

(4) Movement of the drilling cylinder at $\mathrm{c} 1$

When the drilling cylinder piston rod moves to the travel switch $\mathrm{c} 1, \mathrm{YC} 2$ is powered on and the piston rod slows down.

Inlet oil line of the drilling cylinder: oil tank $\rightarrow$ oil hydraulic pump $\rightarrow$ (YC1 electromagnet is powered on, the reversing valve changes the direction) $\rightarrow$ overflow valve $\rightarrow$ left cavity of the drilling cylinder;

Return oil line of the clamping cylinder: left cavity of the clamping cylinder $\rightarrow$ (YC2 electromagnet is powered on, the reversing valve changes the direction) $\rightarrow$ compensated flow control valve $\rightarrow$ oil tank.

Movement of the feeding cylinder and the clamping cylinder remains unchanged.

(5) Movement of the drilling cylinder at c2

When the drilling cylinder piston rod moves to the travel switch $\mathrm{c} 2, \mathrm{YC} 1$ is powered off, $\mathrm{YC} 0$ is powered on, $\mathrm{YC} 2$ is also powered on and the drilling cylinder starts to retreat.

Inlet oil line of the drilling cylinder: oil tank $\rightarrow$ oil hydraulic pump $\rightarrow$ (YC0 is powered on and the reversing valve changes the direction) $\rightarrow$ throttle governing valve $\rightarrow(\mathrm{YC} 2$ is powered on and the reversing valve changes the direction) $\rightarrow$ right cavity of the drilling cylinder;

Return oil line of the drilling cylinder; left cavity of the drilling cylinder $\rightarrow$ overflow valve $\rightarrow$ three-position four-way solenoid valve $\rightarrow$ oil tank.

\section{Full-automatic special drill PLC system} 1) Hardware structure of PLC control system

PLC control system hardware consists of input circuit, output circuit and PLC basic unit ${ }^{[2]}$. Input circuit consists of travel switch, start button and protection switch. To satisfy the above control requirements of the full-automatic special drill, Mitsubishi FX2N-16MR-001 (8 unput/8 point relay output) type PLC is selected.

\section{2) PLC control program under automatic working condition}

In case of PLC control, plan PLC input and output ports first; PLC hardware wiring is shown in Fig. 5:

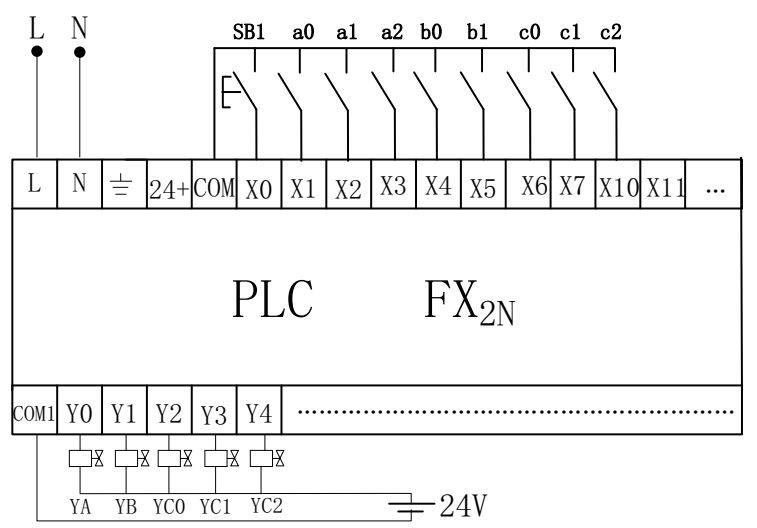

Fig. 5 Full-automatic special drill PLC wiring diagram

According to IO port stated above, PLC ladder diagram is designed as shown in Fig. 6: 


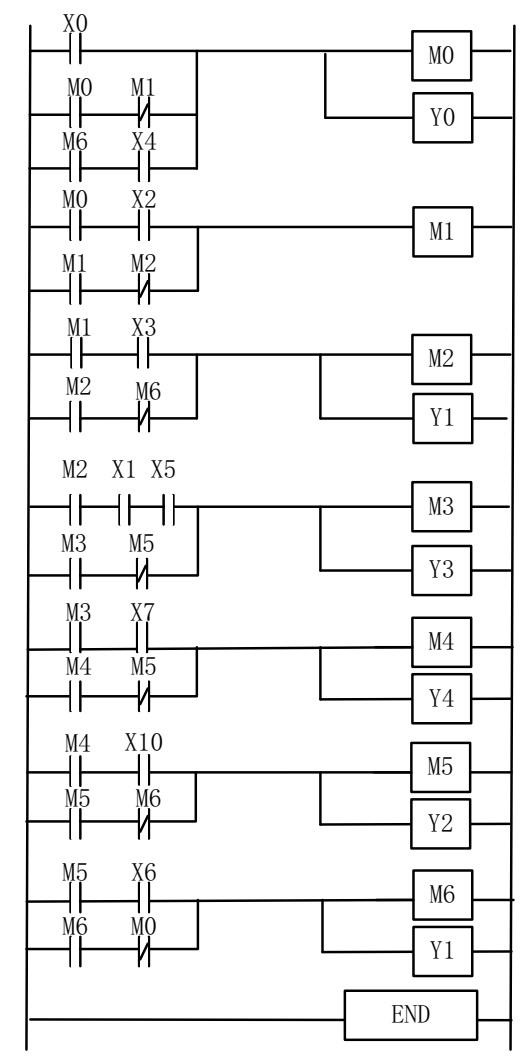

Fig.6 Full-automatic special drill PLC ladder diagram

\section{Conclusions}

Stable performance and good operation of full-automatic special drill demonstrates reasonable and feasible design of the hydraulic system. Full-automatic special drill containing PLC control system greatly lowers the fault rate, improves reliability and automation degree of equipment operation, reduces labor intensity and reaches the expected effect.

References:

[1] Y. X. Lu, Hydropneumatics Manual [M]. Beijing: China Machine Press. (2002. 1.)

[2] J. W. Luo, Hydraulic Drive and Control [M]. Chongqing:

Chongqing University Press. (2006. 7. ) 